\title{
The Effect of Combination Therapy on Mortality and Adverse Events in Patients with Staphylococcus aureus Bacteraemia: A Systematic Review and Meta- analysis of Randomized Controlled Trials
}

\author{
Chao Ye $\cdot$ Chunjiang Wang $\cdot$ Zuojun Li $\cdot$ Xin Li $\cdot$ Juan Pan \\ Liang Liu $\cdot$ Zhaohui Wang
}

Received: August 19, 2021 / Accepted: September 15, 2021 / Published online: October 1, 2021

(C) The Author(s) 2021

\begin{abstract}
Introduction: The findings of randomized controlled trials (RCTs), observational studies, and meta-analyses vary regarding the effectiveness and safety of combination therapy for patients with Staphylococcus aureus bacteraemia (SAB). We aimed to identify the effectiveness and safety of combination therapy in patients with SAB compared with those of monotherapy.
\end{abstract}

Chao Ye and Chunjiang Wang contributed equally to this paper.

Supplementary Information The online version contains supplementary material available at https:// doi.org/10.1007/s40121-021-00539-y.

C. Ye $\cdot$ X. Li

Department of Pharmacy, The Third Hospital of Changsha, Changsha, Hunan, People's Republic of China

C. Wang $\cdot$ Z. Li

Department of Pharmacy, The Third Xiangya

Hospital, Central South University, Changsha,

Hunan, People's Republic of China

J. Pan

Department of Pharmacy, Liuyang Hospital of Traditional Chinese Medicine, Changsha, Hunan, People's Republic of China

L. Liu

Neurosurgery, People's Hospital of Ningxiang City
Methods: We performed a systematic review and meta-analysis to compare combination therapy versus monotherapy in patients with SAB. Two authors independently searched PubMed, Embase, and the Cochrane Library of clinical trials until 17 February 2021. Any RCT comparing mortality or adverse events (AEs) of combination therapy versus monotherapy for patients with SAB was eligible. Summary risk ratios (RRs) and 95\% confidence intervals (CIs) were evaluated using a random-effects model. The primary outcome was all-cause mortality at any time point. This meta-analysis is registered with the PROSPERO database (CRD42020188176) and reported according to PRISMA guidelines.

Results: In total, 1906 articles were identified and screened, and 14 studies (2367 patients)

Affiliated to Hunan University of Chinese Medicine, Changsha, Hunan, People's Republic of China

\section{Z. Wang (ه)}

Department of Pharmacy, People's Hospital of Ningxiang City Affiliated to Hunan University of Chinese Medicine, Changsha, Hunan, People's Republic of China

e-mail: wangzhaohuiry@163.com

\section{Z. Wang}

Department of Pharmacy, People's Hospital of Ningxiang City Affiliated to Hunan University of Chinese Medicine, Yihuan West Road, Ningxiang City 410600, Hunan, People's Republic of China 
were included in the meta-analysis. There was no significant difference in the risk of all-cause mortality between the two groups $(\mathrm{RR}=1.00$; 95\% CI 0.83-1.20; $P=0.99 ; I^{2}=0 \%$ ). Similar results were obtained by subgroup analysis of mortality recording time, endocarditis, pathogen resistance, article publication time, number of patients, and adjuvant antibiotics. Notably, combination treatment might significantly increase the risk of drug-related AEs $(\mathrm{RR}=1.68$; 95\% CI 1.06-2.66; $\left.P=0.03 ; I^{2}=67 \%\right)$ and nephrotoxicity $(\mathrm{RR}=2.30$; 95\% CI $1.68-3.16$; $P<0.00001 ; I^{2}=0 \%$ ), although the occurrences of AEs leading to treatment discontinuation and serious AEs were not significantly different between the two groups.

Conclusions: The meta-analysis suggested that combination therapy could not reduce mortality but might increase the risk of drug-related AEs and nephrotoxicity and should be applied very cautiously. Future studies on combined drug therapy for $\mathrm{SAB}$ need careful and rigorous design for specific antibiotic combinations.

Keywords: Staphylococcus aureus; Bacteraemia; Combination therapy; Mortality; Meta-analysis

\section{Key Summary Points}

Fourteen eligible RCTs involving 2367 patients were included for the final analysis

Meta-analyses showed that there was no significant difference in the risk of allcause mortality between combination therapy and monotherapy groups

Combination therapy may increase the risk of nephrotoxicity and drug-related AEs, which will limit its clinical application

Future studies on combined drug therapy for $\mathrm{SAB}$ need careful and rigorous design for specific antibiotic combinations

\section{INTRODUCTION}

Staphylococcus aureus is among the leading causes of infections in hospital- and communityacquired infection patients and is associated with significant morbidity. Enhanced infection control efforts have helped reduce the rate of $S$. aureus bacteraemia (SAB) over the past decade, but case fatality rates for SAB have improved only modestly in recent decades. During this time, the incidence of SAB in the general population has ranged from 20 to 25 episodes per $10^{5}$ patient-years, with mortality rates of approximately 20-30\% [1-4].

Despite straightforward evidence-based combination interventions for $\mathrm{SAB}$, doubts remain regarding the best antimicrobial therapy [5]. For decades, treatment has included antistaphylococcal penicillins (ASPs) or first-generation cephalosporins (such as cefazolin) for methicillin-sensitive $S$. aureus bacteraemia (MSSAB) and vancomycin or daptomycin for methicillin-resistant $S$. aureus bacteraemia (MRSAB) $[6,7]$. Due to the potential severity of $\mathrm{SAB}$, there remains active interest in novel treatment strategies to improve clinical outcomes.

In vitro laboratory data, animal models, and observational studies have suggested that combination antimicrobial therapy may be beneficial for S. aureus [8-12]. Over the past several years, there has been an increase in the number of randomized controlled trials (RCTs) involving patients with $\mathrm{SAB}$, including the use of adjunctive rifampicin, fosfomycin, or ceftaroline for $S A B$ [13-16]; however, the evidence supporting combination therapy in $\mathrm{SAB}$ is unclear. Several efforts have been made through meta-analyses to investigate whether combination antibiotic therapy is associated with better clinical outcomes than monotherapy. However, these meta-analyses either included a small number of patients with $S$. aureus infections or most of the included studies were non-RCTs with large heterogeneity that affected the reliability of the results [17-19]. We aimed to systematically review and synthesize the published RCTs via meta-analysis methodology to examine whether combination antibiotic therapy 
was associated with more favourable clinical outcomes than monotherapy in patients with SAB.

\section{METHODS}

\section{Information Sources and Key Word Search}

Using the PubMed, Embase, and Cochrane databases, searches for relevant articles were performed with the following items: 'Staphylococcus aureus' and 'bacteraemia or septicaemia or bloodstream infection' and 'randomized controlled trial'. The search used a combination of subject words and free words. Searches were limited to articles published in English up to 17 February 2021. References from clinical trials were searched manually to identify potentially relevant studies. The review protocol is registered at the Prospero international prospective register of systematic reviews (registration no. CRD42020188176), and the article is reported according to PRISMA guidelines.

\section{Inclusion and Exclusion Criteria}

Only full-length published trials in adult patients with SAB were included in the analysis. Studies were included if they met the following criteria. (1) They compared the efficacy or safety of treatment between monotherapy and combination therapy in two groups of patients. Usually, monotherapy was defined as therapy with ASPs or first-generation cephalosporins for MSSAB. If the patients were allergic to the above agents, clindamycin, vancomycin, or daptomycin was used as alternative treatment; vancomycin or daptomycin was used for MRSAB. Any one of the above primary drugs plus another antibiotic was considered combination therapy. (2) The included studies were all RCTs. (3) At least one of the following outcomes was reported: mortality and adverse effects (AEs). Articles were excluded if outcomes were not reported, outcome data were not readily extractable for each treatment group, or the article was not available in English.

\section{Data Extraction and Quality Assessment}

Two researchers independently reviewed the included studies and extracted the relevant information from each study. Disagreement between the two reviewers was resolved by discussion until consensus was reached. We used a standard form to extract relevant data from the included articles, including author name, study period, type of pathogen, study country, publication year, patient population, characteristics of bacteraemia, data relevant to therapy (antimicrobials used), mortality and AE outcome, and numbers of different groups. Risk of bias and study quality were assessed using the Cochrane Risk of Bias 2 tool [20]. This was applied to each study independently by two reviewers, with disagreements resolved by involvement of a third reviewer [20].

\section{Outcomes and Definitions}

The primary outcome was all-cause mortality. If multiple time points were reported, mortality at the latest time point (i.e., reporting the highest number of deaths) was included in the main analysis. When mortality was provided for both the intention-to-treat (ITT) and the per-protocol populations, we used the ITT population. Only if ITT data were not available did we include per-protocol data in the meta-analysis. The safety outcomes included any adverse event (AEs), any serious adverse events (SAEs), drugrelated AEs, and any AEs leading to treatment discontinuation and nephrotoxicity. These definitions were the same as the definitions described in each publication.

\section{Statistical Methods}

Data were analysed using Review Manager (RevMan 5.3; The Nordic Cochrane Centre, Cochrane Collaboration, 2008). We calculated pooled risk ratios (RRs) and 95\% confidence intervals (CIs) using the Mantel-Haenszel random-effects model and considered a $P$ value $<$ 0.05 to be statistically significant $[21,22]$. Statistical heterogeneity among studies was assessed by $\chi^{2}$ test $(P<0.10$ indicated significant 
heterogeneity) and $I^{2}$. RevMan or STATA software (version 15.1; Stata Corp., University City, TX, USA) was used to create a funnel chart to assess publication bias. Publication bias of the included studies was analysed using Egger's test [23]. A $P$ value $\leq 0.05$ for any of those tests was indicative of the presence of bias. We performed subgroup analyses to assess the effect of mortality recording time, endocarditis, pathogen resistance, article publication time, number of patients, and adjuvant antibiotics on the primary outcome.

\section{Compliance with Ethics Guidelines}

This article is based on previously conducted studies and does not contain any new studies with human participants or animals performed by any of the authors.

\section{RESULTS}

\section{Study Selection and Characteristics}

As shown in Fig. 1, the literature search strategy initially identified 1906 articles (390 from PubMed, 917 from EMBASE, 596 from The Cochrane Library, and 3 from other sources). Five hundred ninety-seven studies were excluded because they were duplicated studies. A total of 1261 articles were removed after initial screening of titles and abstracts. Of the remaining 48 studies, we excluded 34 studies from the full-text evaluation for five main reasons. In total, after the full-text screening, we included 14 RCTs [13-16, 24-33] in the qualitative synthesis. Of these 14 studies, 9 were multicentre studies [13-16, 24-27, 30] and 5 were single-centre studies [28, 29, 31-33]. Only one of the nine multicentre studies was transnationally conducted in Australia, Singapore, Israel, and New Zealand [14]. Of the 13 non-transnational studies, five were from the USA, six were from Europe (two from Spain, two from Belgium, one from England and one from Finland), one was from Canada, and one was from Australia. Five of the 14 studies evaluated only MRSAB [14-16, 25, 29] and MSSAB
$[24,27,28,32,33]$, while the other 4 studies included both MRSAB and MSSAB $[13,26,30,31]$. Standard therapy varied; ASPs were usually the most commonly used agent for MSSAB, whereas others included cefazolin and clindamycin, and vancomycin or daptomycin was the main agent for MRSAB. The adjuvant agents in the combined treatment group also varied, with gentamicin as an additive in four studies [26, 28, 32, 33], rifampicin as an additive in four studies [13, 29-31], and daptomycin [24], levofloxacin [27], fosfomycin [15], or ceftaroline [16] as an additive in one study. In the other two studies, ASPs or cefazolin was used as additives [14, 25]. Other characteristics included in the study, such as the age of the patients, the definition of outcome indicators, and the number of patients in the two groups, are shown in Table 1 . The source of infection sites of the two groups, Charlson comfort index, and Sequential Organ Failure Assessment (SOFA) score are shown in Table S1.

\section{Quality Assessment}

Two independent review authors independently assessed the risk of bias for each of the included studies and reached agreement. Figures 2 and 3 also provide a summary of the risk of bias in all included studies. Eleven trials described an adequate random sequence generation process with low risks for selection bias. Nine trials described the methods used for allocation concealment with low risks for selection bias. Three trials were designed in a double-blind model with low risks for performance bias. Four trials were designed with blinding of outcome assessment with low risks for detection bias. We determined that incomplete outcome data (attrition bias) introduced a low risk of bias in eight studies. We determined that seven of the included studies had a low risk of reporting bias, as the study protocols were available, and all outcomes of interest had been reported in the prespecified way. 


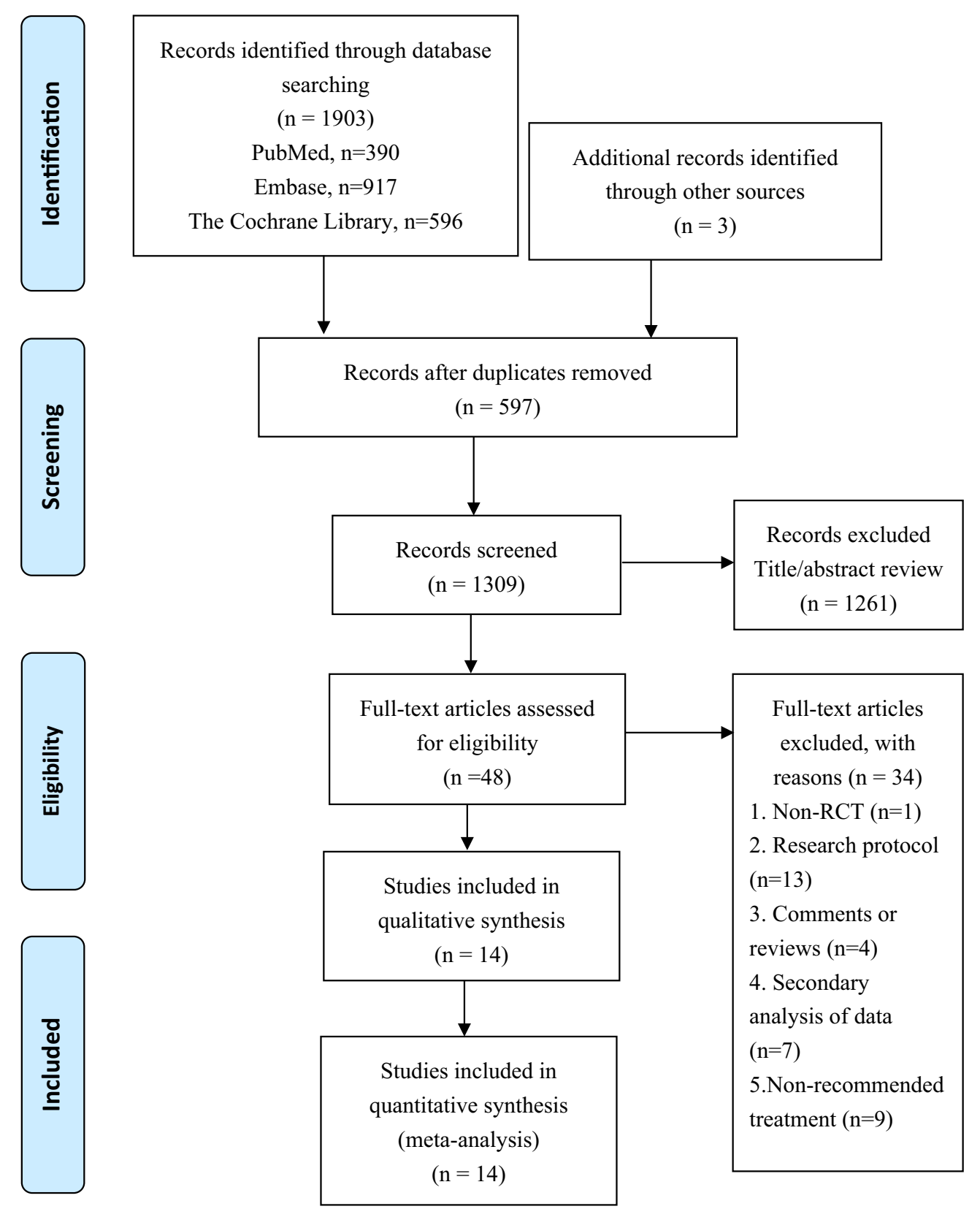

Fig. 1 Flow diagram of the selection of studies for inclusion in the meta-analysis

\section{Primary Outcome}

A meta-analysis of the 14 studies including 2360 patients suggested that there was no significant difference in the rate of all-cause mortality between the two groups ( $\mathrm{RR}=1.00 ; 95 \% \mathrm{CI}$ 0.83-1.20; $P=0.99 ; I^{2}=0 \%$ ) (Fig. 4). Subgroup analysis also suggested that there was no significant difference in all-cause mortality between the two groups at 7, 14, 30, 42, 60, and 90 days (Figure S1). Subgroup analysis of pathogen resistance suggested that there was no significant difference in mortality between the two groups, whether the infecting pathogen was MSSA $\quad(\mathrm{RR}=0.99 ; \quad 95 \%$ CI $0.79-1.24$; 


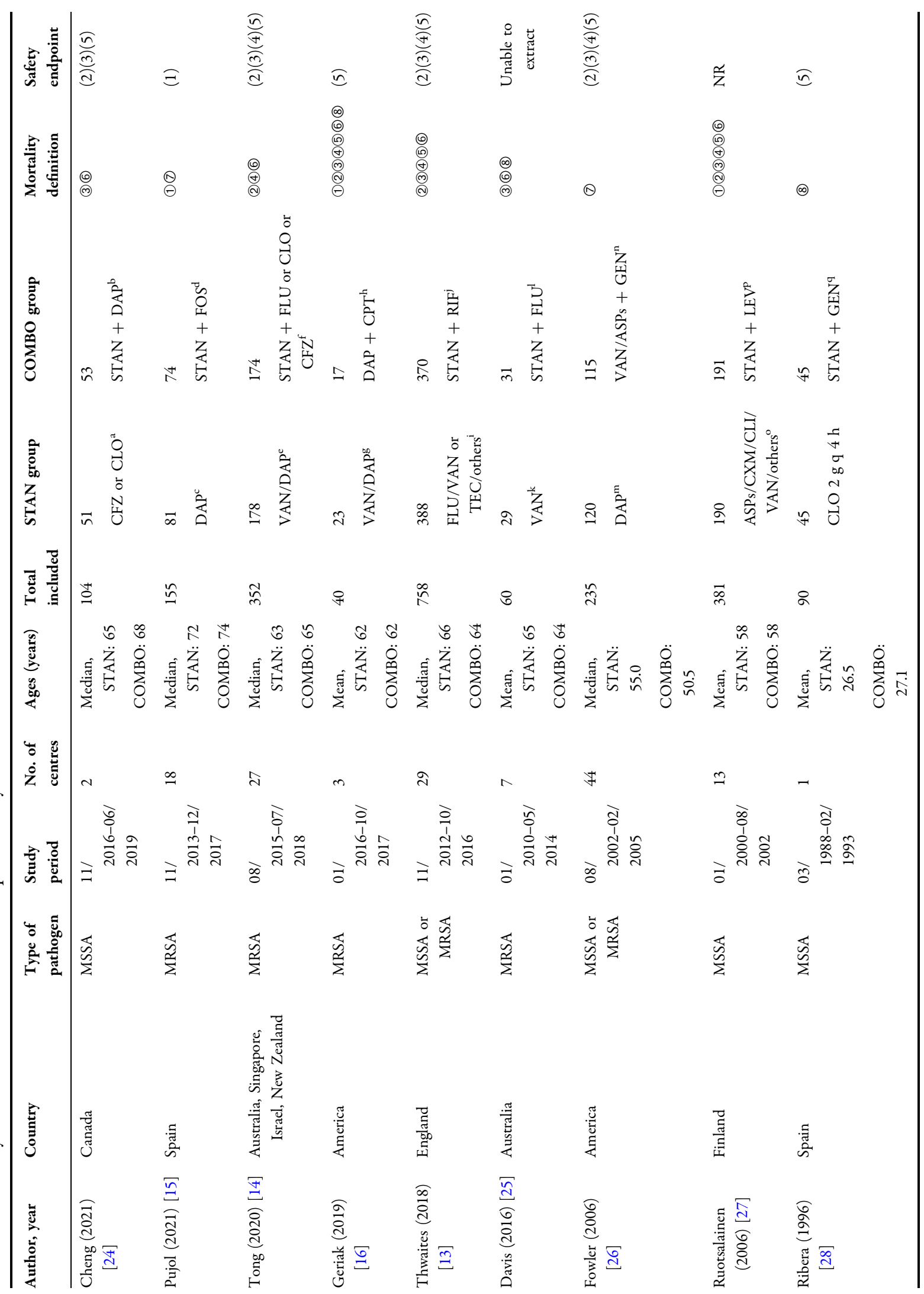




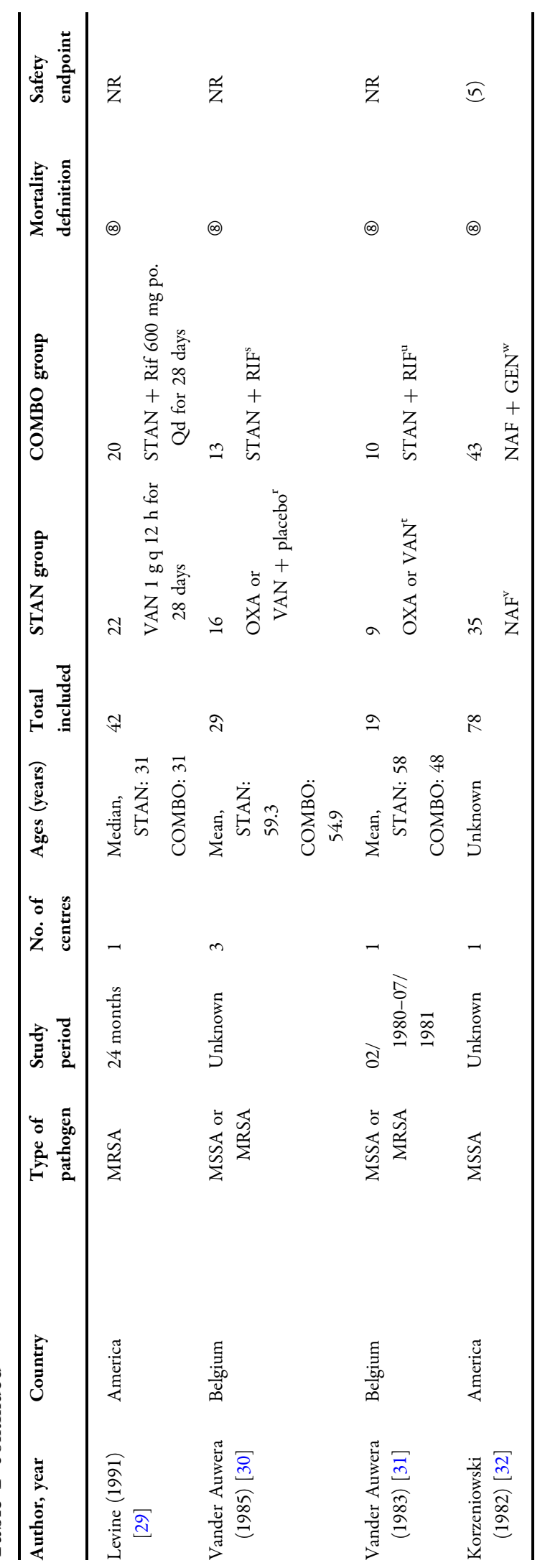




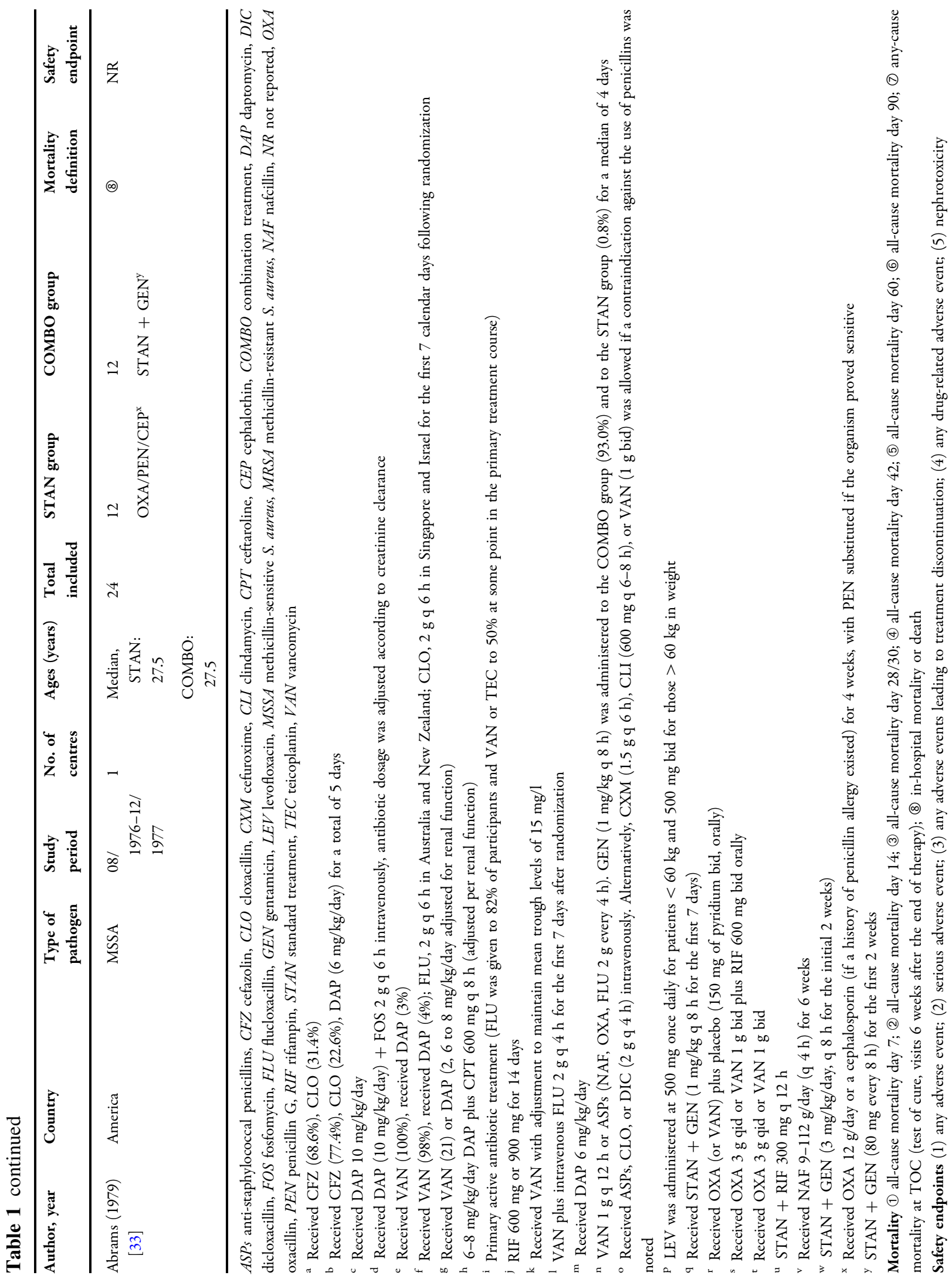




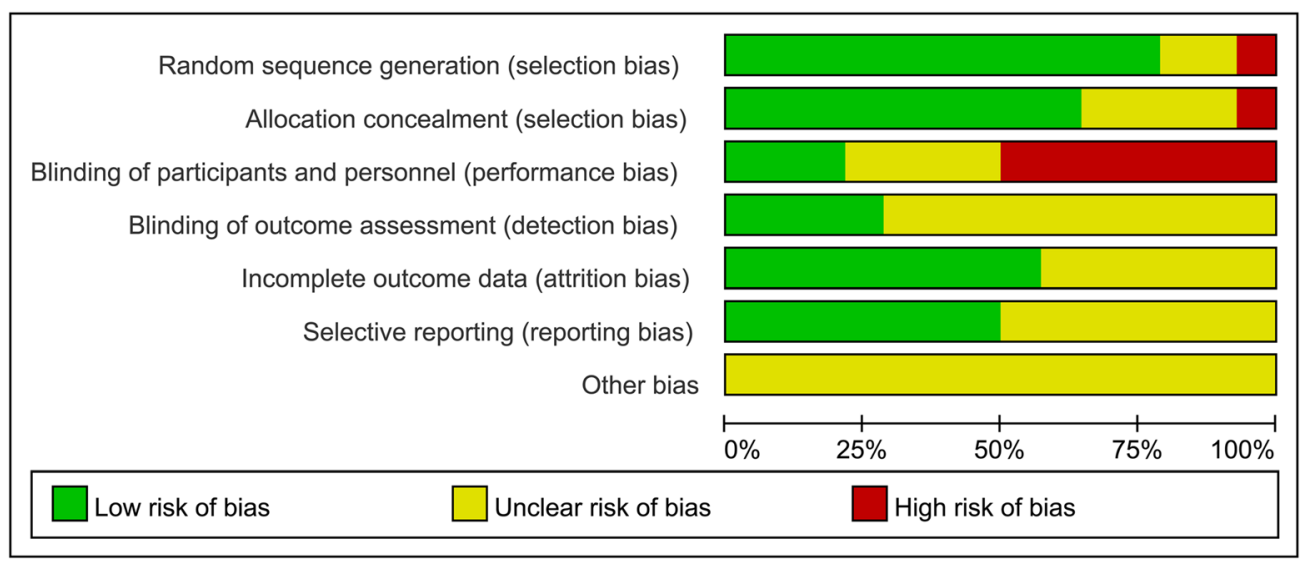

Fig. 2 Risk of bias graph: review authors' judgement about each risk of bias item presented as percentages across all included studies

$\left.P=0.93 ; I^{2}=0 \%\right)$ or MRSA $(\mathrm{RR}=0.88 ; 95 \% \mathrm{CI}$ $0.53-1.47 ; P=0.62 ; I^{2}=27 \%$ ) (Fig. 5). Finally, no statistically significant difference was found in all-cause mortality between the compared treatment arms when the five studies that examined patients with $S$. aureus endocarditis were analysed $(\mathrm{RR}=1.11 ; 95 \%$ CI $0.62-1.99$; $P=0.72 ; I^{2}=0 \%$ ) (Fig. 6).

Other factors that may affect the robustness of the outcome were also analysed by subgroups. The results of the subgroup analysis of the publication year showed that there was also no significant difference in mortality between the two groups (studies published before 2006, $\mathrm{RR}=0.89 ; 95 \%$ CI $0.43-1.86 ; P=0.76 ; I^{2}=4 \%$; studies published in or after 2006, RR $=1.01$; 95\% CI 0.84-1.22; $\left.P=0.93 ; I^{2}=0 \%\right)$. Similarly, the results of subgroup analysis to assess the size of the study population also yielded similar results (number of patients $\geq 200, \mathrm{RR}=1.05$; 95\% CI $0.85-1.30 ; P=0.66 ; I^{2}=0 \%$; number of patients $<200, \mathrm{RR}=0.87 ; 95 \%$ CI $0.61-1.25$; $\left.P=0.46 ; I^{2}=0 \%\right)$. The results of other subgroup analyses showed no significant difference in mortality between the two groups (Table 2).

\section{Safety Outcome}

A meta-analysis of the three studies including 1154 patients suggested that there was no significant difference in the rate of any SAEs between the two groups $(\mathrm{RR}=0.97,95 \% \mathrm{CI}$ $0.80-1.18 ; P=0.78 ; I^{2}=19 \%$ ) (Figure S2). A meta-analysis of 1450 patients in four studies suggested that the combination treatment group had a higher incidence of drug-related AEs than the monotherapy group $(\mathrm{RR}=1.68$; 95\% CI 1.06-2.66; $P=0.03 ; I^{2}=67 \%$ ) (Fig. 7). A meta-analysis of 500 patients in three studies suggested that there was no significant difference in the rate of any AEs leading to treatment discontinuation between the two groups $(\mathrm{RR}=$ 1.76; 95\% CI $0.72-4.32 ; \quad P=0.21 ; I^{2}=36 \%$ ) (Figure S3).

A meta-analysis of seven studies including 1580 patients suggested that combination therapy significantly increased the risk of nephrotoxicity compared with that for monotherapy $(\mathrm{RR}=2.30$; 95\% CI 1.68-3.16; $P<0.00001 ; I^{2}=0 \%$ ) (Fig. 8).

\section{Publication Bias}

A funnel chart for the main outcome indicator of all-cause mortality and the publication bias test revealed a basically symmetrical left and right side of the funnel chart, and combined with Egger's test results (bias, - 0.39; 95\% CI -1.39 to $0.61 ; P=0.411$ ), these findings suggested a small possibility of publication bias (Fig. 9). 


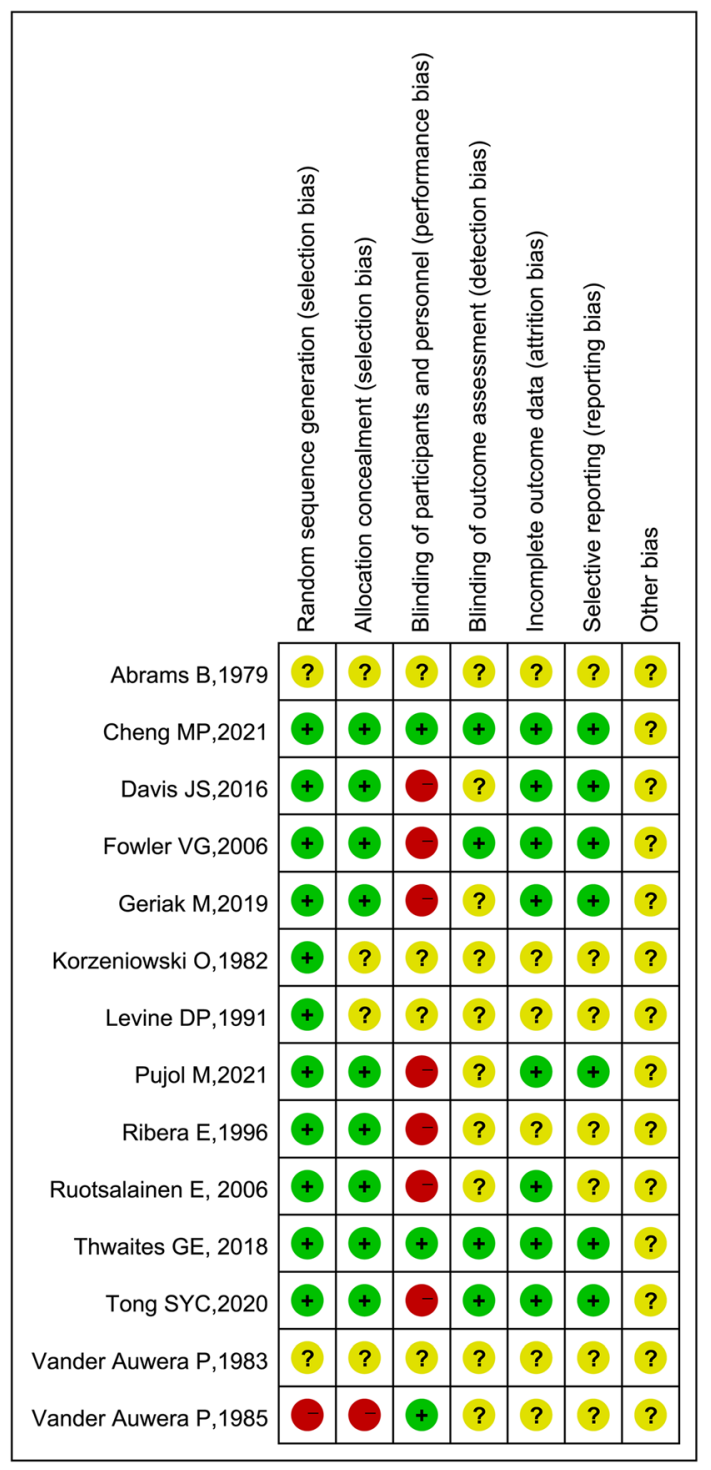

Fig. 3 Risk of bias summary: review authors' judgement about each risk of bias item for each included study

\section{DISCUSSION}

This meta-analysis summarizes the best available evidence of the administration of combination antibiotic therapy vs. monotherapy for treating SAB. Compared with monotherapy, the combination treatment, regardless of the resistance of the pathogenic bacteria resistance, did not significantly reduce the short- and longterm mortality of patients with SAB. The analysis of patients with $S$. aureus endocarditis and other evaluable subgroups was consistent with the main results. With respect to safety, although there was no significant difference in the incidence of SAEs or any AEs leading to treatment discontinuation between the two groups, the combination treatment group had more drug-related AEs and nephrotoxicity than monotherapy.

In terms of both clinical burden and absolute number of patients, SAB remains a common and highly morbid infection $[34,35]$. The emergence of antibiotic resistance and continued high failure rate, including unacceptable mortality, in patients receiving standard therapies for $\mathrm{SAB}$ demonstrate the need for new therapeutic agents and approaches to this disease.

Improved outcomes for patients with $\mathrm{SAB}$ have been sought through efforts to enhance antimicrobial efficacy by using combination antimicrobial therapy. In a previous metaanalysis of $\beta$-lactams combined with aminoglycosides, rifampicin combined with standard regimens for the treatment of $\mathrm{SAB}, \beta$-lactams combined with vancomycin, or daptomycin in the treatment of MRSAB, it was suggested that combination therapy cannot significantly reduce the mortality of patients compared with that for monotherapy $[17,18,36]$. The above three meta-analyses are consistent with our results; however, owing to the poor quality and heterogeneity of the studies included in these meta-analyses, convincing clinical data are sparse. Compared with previously published works, this meta-analysis is not limited by the inclusion of non-randomized studies with heterogeneity and confounders. To our knowledge, this study included the largest number of patients from geographically diverse regions. Additionally, almost all the different combination therapies used in the clinic were included in our study.

We performed several sensitivity analyses focusing on more homogeneous subsets of patients, such as those with bacterial endocarditis, to further evaluate our main study question. The results of subgroup analysis also suggested that rifampicin or gentamicin as an adjuvant combined with standard agents did not significantly reduce mortality compared 


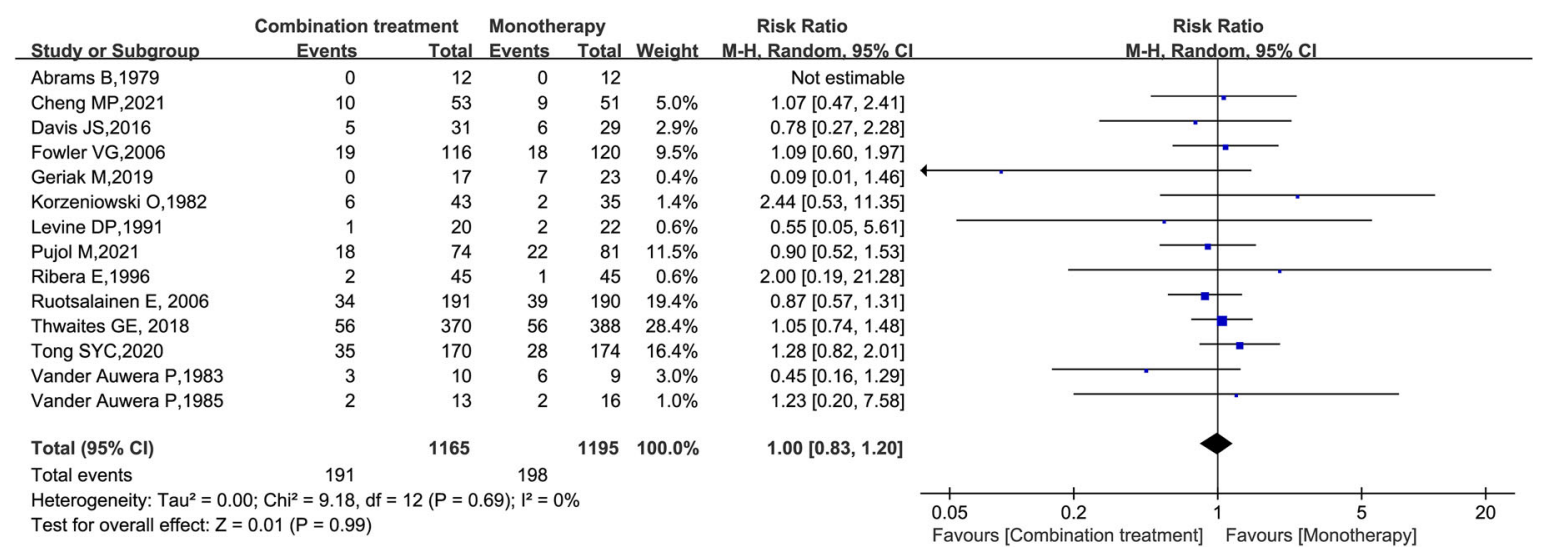

Fig. 4 Forest plot of the risk ratio (RR) for all-cause mortality in patients with Staphylococcus aureus bacteraemia (SAB)

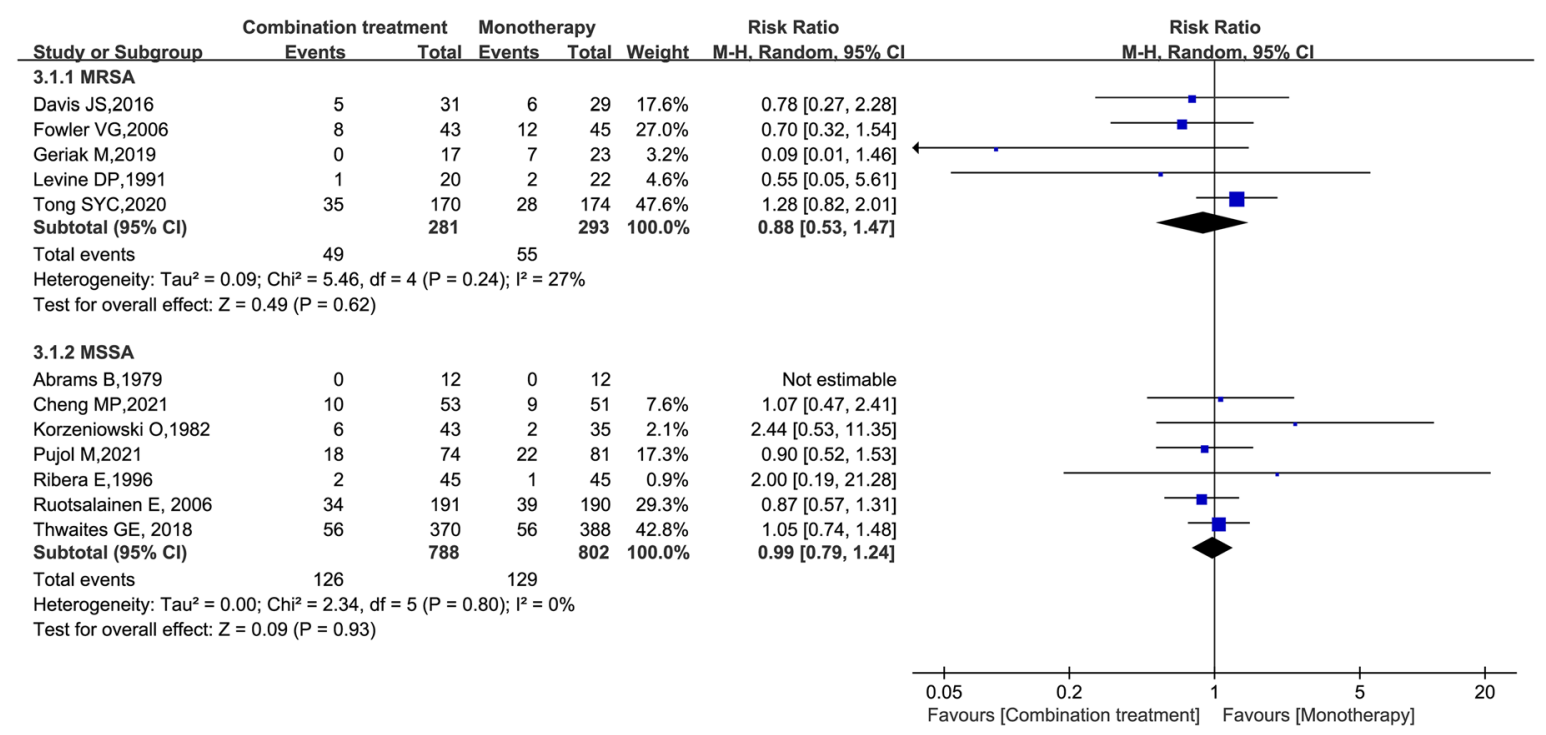

Fig. 5 Forest plot of the risk ratio (RR) for all-cause mortality in patients with methicillin-sensitive $S$. aureus bacteraemia (MSSAB) or methicillin-resistant S. aureus bacteraemia (MRSAB)

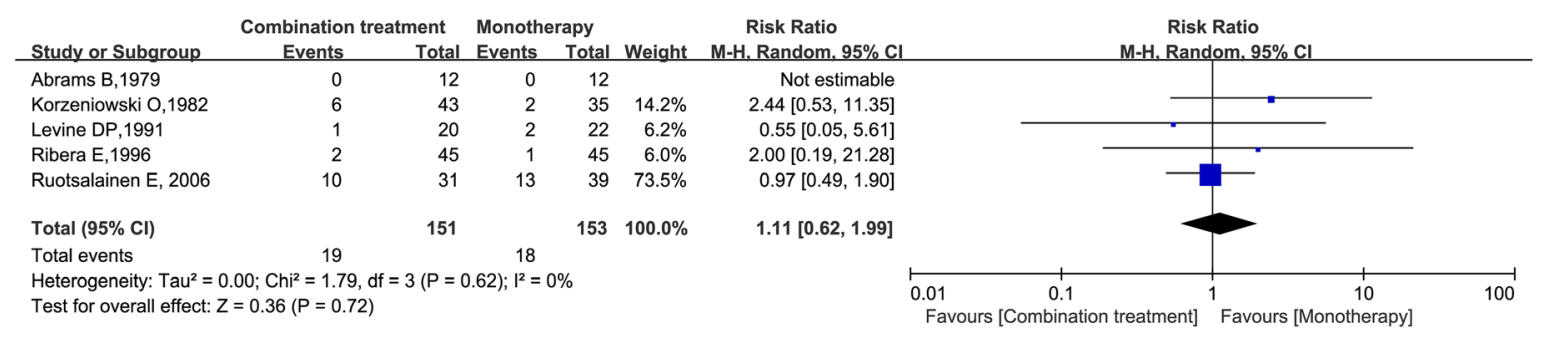

Fig. 6 Forest plot of the risk ratio (RR) for all-cause mortality in patients with $S$. aureus endocarditis 
Table 2 Subgroup analysis of the effect on all-cause mortality

\begin{tabular}{lllllll}
\hline Subgroup & No. of studies & $\begin{array}{l}\text { COMBO } \\
\text { (events/total) }\end{array}$ & $\begin{array}{l}\text { STAN } \\
\text { (events/total) }\end{array}$ & Risk ratio (95\% CI) & $\boldsymbol{P}$ value $^{\boldsymbol{I}^{2} \%}$ \\
\hline $\begin{array}{l}\text { Year of publication } \\
\text { Before 2006 }\end{array}$ & 6 & $14 / 143$ & & & & \\
In or after 2006 & 8 & $177 / 1022$ & $185 / 1056$ & $1.01(0.84-1.22)$ & 0.93 & 0 \\
Number of patients & & & & & \\
$\geq 200$ & 4 & $144 / 847$ & $141 / 872$ & $1.05(0.85-1.30)$ & 0.66 & 0 \\
$<200$ & 10 & $47 / 318$ & $57 / 323$ & $0.87(0.61-1.25)$ & 0.46 & 0 \\
Adjuvant agents & & & & & & \\
BLs & 2 & $40 / 201$ & $34 / 203$ & $1.19(0.78-1.80)$ & 0.42 & 0 \\
Fosfomycin & 1 & $18 / 74$ & $22 / 81$ & $0.90(0.52-1.53)$ & 0.69 & - \\
Daptomycin & 1 & $10 / 53$ & $9 / 51$ & $1.07(0.47-2.41)$ & 0.87 & - \\
Ceftaroline & 1 & $0 / 17$ & $7 / 23$ & $0.09(0.01-1.46)$ & 0.09 & - \\
Rifampin & 5 & $102 / 678$ & $87 / 501$ & $0.66(0.37-1.19)$ & 0.17 & 66 \\
Levofloxacin & 1 & $34 / 191$ & $39 / 190$ & $0.87(0.57-1.31)$ & 0.50 & - \\
Gentamicin & 4 & $27 / 216$ & $21 / 212$ & $1.24(0.73-2.13)$ & 0.43 & 0 \\
\hline
\end{tabular}

STAN standard treatment, COMBO combination treatment, BLs $\beta$-lactam antibiotics

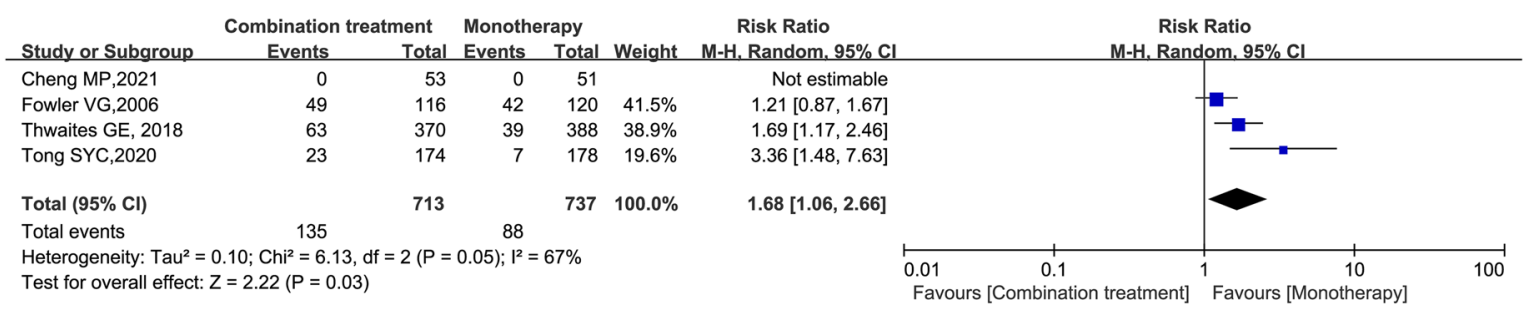

Fig. 7 Forest plot of the risk ratio (RR) for drug-related adverse events in patients with $S$. aureus bacteraemia (SAB)

with that for the standard regimen alone, which is consistent with the recommendation of the Infectious Diseases Society of America (IDSA) in 2011 that rifampicin or gentamicin combined with vancomycin is not recommended for the treatment of MRSAB or natural valve endocarditis [34]. Therefore, our results further confirm that the evidence available from the trials analysed does not show any survival benefit for the addition of synergistic drugs to a standard treatment of patients with SAB. In contrast, such an addition can lead to increased nephrotoxicity and drug-related AEs.

At present, many clinical studies and several meta-analyses have suggested that combining antibacterial agents could reduce the risk of microbial failure, recurrence of bacteraemia, and persistent bacteraemia and shorten the duration of bacteraemia in patients with $\mathrm{SAB}$ [13-15, 18, 19, 25, 37-43]. However, translating 


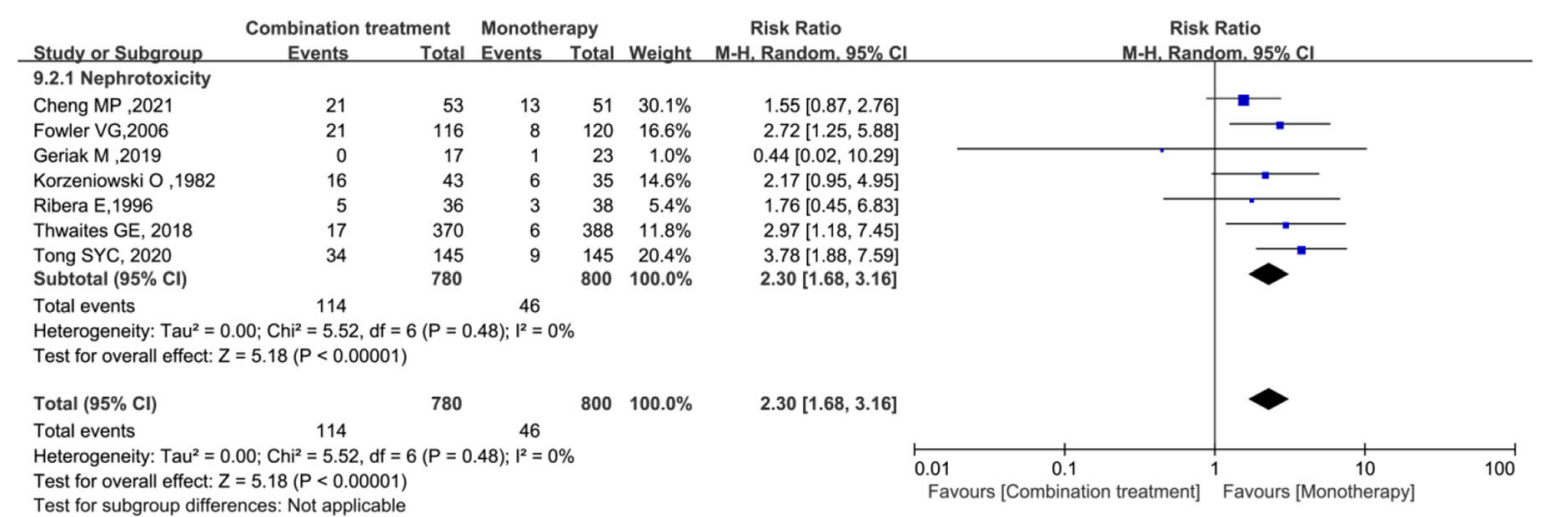

Fig. 8 Forest plot of the risk ratio (RR) for nephrotoxicity in patients with $S$. aureus bacteraemia (SAB)

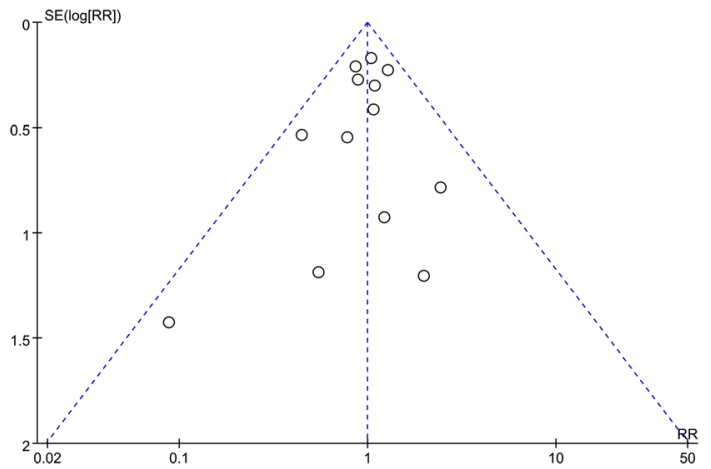

Fig. 9 Funnel chart for all-cause mortality indicators by RevMan

clinical success to survival in critically ill patients with $S A B$ may be difficult and requires in-depth knowledge of the mechanisms and possible confounders contributing to death.

First, combined antimicrobial therapy can synergize sterilization and prevent the emergence of drug resistance, but it also leads to an increase in AEs, which leads to the elimination of the clinical survival benefits provided by combination therapy. An international randomized trial included in our meta-analysis was stopped by the data and safety monitoring board because of apparent futility, as evidenced by the absence of an effect on the composite outcome of death or complications by day 90 , together with an imbalance in acute kidney injury (AKI) [14]. In this study, AKI occurred in
$23.4 \%$ of combination therapy recipients but in only $6.2 \%$ of subjects receiving monotherapy (risk difference 17.2\%; 95\% CI 9.3-25.3; $P=0.001$ ); a greater proportion of the AKI in the combination therapy group was of a higher severity, and the 45-day all-cause mortality of the two groups of patients was $15 \%$ and $11 \%$, respectively. In another large (744 participants) multicentre, randomized, double-blind placebocontrolled trial, the effort to harness the potent anti-staphylococcal activity of rifampicin by combining it with standard antibiotic therapy for $\mathrm{SAB}$ failed to reduce mortality or bacteriological failure compared to those for standard of care plus placebo $(17 \%$ vs. $18 \%$; absolute risk difference $-1.4 \%$; $95 \%$ CI -7.0 to 4.3 ; $P=0.81)$ [13]. In contrast, a trend towards increased occurrence of drug-modifying AEs (17\% vs. $10 \% ; P=0.004)$ and renal grade $3-4$ AEs (5\% vs. $2 \% ; P=0.053)$ was noted among rifampicin recipients, as was a significant increase in the occurrence of drug-drug interactions in this study ( $6 \%$ vs. $2 \% ; P=0.0005)$. A recent systematic review involving $>2$ million participants showed that individuals with AKI were at increased long-term risk for chronic kidney disease, end-stage kidney disease, and death [44]. In this meta-analysis, compared with monotherapy, combination therapy significantly increased the incidence of drug-related AEs and nephrotoxicity, which may explain why combination antibiotics did not provide survival benefits for SAB patients. 
Furthermore, combination therapy may have survival benefits for patients with complicated and metastatic bacteraemia with higher mortality, and only some specific combination therapy with less toxicity and more synergistic sterilization can reduce mortality $[16,45]$. In contrast to other $\beta$-lactam antibacterial drugs, ceftaroline is active against MRSA by binding to PBP2A and inhibiting peptidoglycan transpeptidation. Similar to other $\beta$-lactams, ceftaroline enhances daptomycin cell membrane binding. Therefore, ceftaroline combined with vancomycin or daptomycin is theoretically a more effective combination than other combination regimens for patients with MRSAB. A randomized controlled open-label pilot study compared the primary treatment of MRSAB with daptomycin plus ceftaroline with vancomycin. This study was terminated early when an unanticipated mortality difference was noted between the groups: $0 / 17$ in the combination arm vs. $6 / 23$ among vancomycin recipients $(P=0.029)$. Moreover, there were minimal treatment-related AEs [16]. Another large retrospective matched cohort study (171 participants) compared patients with MRSA bacteraemia treated with standard of care (SOC) treatment versus daptomycin plus ceftaroline. Many of the combination patients were inherently high risk because the combination was used in a salvage role, but despite this consideration, daptomycin-ceftaroline resulted in a numerically lower 30 -day mortality rate than SOC $(8.3 \%$ vs. $14.2 \%$ ) [46]. However, two recent retrospective cohort studies evaluated the efficacy of the combination of ceftaroline with vancomycin or daptomycin in the treatment of patients with complicated MRSAB. Disappointingly, compared with monotherapy, combination therapy failed to reduce mortality $[47,48]$. It is worth noting that the small sample size and inherent biases in clinician choice for selecting combination treatment, whereby those receiving combination treatment are likely at higher risk of treatment failure, should be cautiously interpreted. At present, the contradictory survival benefit results, high cost, and unavailability in most areas necessitate careful implementation of the combination treatment regimen containing ceftaroline without the support of more powerful large-scale randomized controlled trials.

For the management of $\mathrm{SAB}$, reasonable and timely administration of antibacterial drugs is very important, but it is only one important part of many measures. Comprehensive management of SAB involves timely identification of the infecting strain and source of infection, proper choice of antibiotic treatment, and robust prevention strategies [49]. The combination of antibiotics plus a comprehensive clinical approach to managing MRSAB may have a positive impact on patient outcomes. Recently, a 7-year retrospective, quasi-experimental study involving 813 adult MRSAB patients suggested that compared with prepathway (PRE) patients $(n=379)$, those treated postpathway (POST, $n=434$ ) plus combination treatment (vancomycin or daptomycin combined with $\beta$-lactams) had a significant reduction in 30 -day $(9.7 \%$ vs. $15.6 \%$, $P=0.011)$ and 90 -day mortality $(12.2 \%$ vs. 19.0\%, $P=0.007$ ) [50]. Moreover, compared with that in the PRE group, the incidence of AKI in the POST group was similar $(9.6 \%$ vs. $7.2 \%$, $P=0.282$ ). Notably, most of the adjunctive agents in the combined treatment of this study were cefazolin or cefepime, which avoided the use of ASPs with higher nephrotoxicity [51, 52].

Several limitations should be taken into consideration when interpreting the results of this meta-analysis. These studies included patients with primary bacteraemia or bacteraemia secondary to different infection sites, so employing either monotherapy or combination therapy should be considered depending on the type of infection (e.g., pneumonia, catheter infection, and urinary tract infection). Moreover, we found that very few studies have analysed the impact of possible confounding factors on the mortality of patients in different treatment groups, including the SOFA score, Charlson comorbidity index, age groups, and origin of $S$. aureus infection (community associated or healthcare associated), and data were not available for meaningful comparisons in these subpopulations. Furthermore, although we performed subgroup analysis of adjuvant drugs in the combined treatment regimen, there were too few evaluable studies on 
adjuvant drugs other than gentamicin or rifampicin to further evaluate the efficacy and safety of specific adjuvant drugs. It should not be neglected that in this meta-analysis we included comparative trials to examine special patients with endocarditis. For the treatment of endocarditis, whether patients have value replacement surgery will affect the length of antimicrobial treatment and the prognosis of patients. However, due to the limited data, we cannot further evaluate its impact. Finally, only studies published in English were included. This choice could introduce language bias, which may reduce the accuracy of the combined estimates of the treatment effects.

\section{CONCLUSION}

In conclusion, this meta-analysis suggests that in patients with $\mathrm{SAB}$, the choice between combination therapy and monotherapy does not affect mortality rates. In contrast, compared with monotherapy, combination treatment will significantly increase the occurrence of drugrelated AEs and nephrotoxicity. Therefore, the results of our current meta-analysis do not support routine combination therapy in patients with $\mathrm{SAB}$ as further studies are warranted to evaluate specific antibiotic combinations.

\section{ACKNOWLEDGEMENTS}

Funding. This study was funded by the Hunan Provincial Natural Science Foundation (grant no. 2021JJ80083). The journal's Rapid Service Fee was also funded by the sponsor. The funder had no role in study design, data collection, and analysis, decision to publish, or preparation of the manuscript.

Authorship. All named authors meet the International Committee of Medical Journal Editors (ICMJE) criteria for authorship for this article, take responsibility for the integrity of the work as a whole, and have given their approval for this version to be published.
Authors' Contributions. Substantially contributed to conception or design: Chao Ye, Chunjiang Wang, Zhaohui Wang. Contributed to acquisition, analysis, or interpretation of data: Chao Ye, Xin Li, Juan Pan, Zuojun Li. Drafted the manuscript for important content: Chao Ye, Chunjiang Wang, Zuojun Li. Critically revised the manuscript for important intellectual content: Zhaohui Wang, Liang Liu, Xin Li. Gave final approval: All authors.

Disclosures. Chao Ye, Chunjiang Wang, Zuojun Li, Xin Li, Juan Pan, Liang Liu, and Zhaohui Wang have nothing to disclose.

Compliance with Ethics Guidelines. This article is based on previously conducted studies and does not contain any new studies with human participants or animals performed by any of the authors.

Data Availability. All data generated or analyzed during this study are included in this published article/as supplementary information files.

Open Access. This article is licensed under a Creative Commons Attribution-NonCommercial 4.0 International License, which permits any non-commercial use, sharing, adaptation, distribution and reproduction in any medium or format, as long as you give appropriate credit to the original author(s) and the source, provide a link to the Creative Commons licence, and indicate if changes were made. The images or other third party material in this article are included in the article's Creative Commons licence, unless indicated otherwise in a credit line to the material. If material is not included in the article's Creative Commons licence and your intended use is not permitted by statutory regulation or exceeds the permitted use, you will need to obtain permission directly from the copyright holder. To view a copy of this licence, visit http://creativecommons.org/licenses/bync/4.0/. 


\section{REFERENCES}

1. Tong SYC, Davis JS, Eichenberger E, et al. Staphylococcus aureus Infections: epidemiology, pathophysiology, clinical manifestations, and management. Clin Microbiol Rev. 2015;28(3):603-61.

2. Kourtis AP, Hatfield K, Baggs J, et al. Vital signs: epidemiology and recent trends in methicillin-resistant and in methicillin-susceptible Staphylococcus aureus bloodstream infections-United States. MMWR Morb Mortal Wkly Rep. 2019;68(9):214-9.

3. Simor AE, Pelude L, Golding G, et al. Determinants of outcome in hospitalized patients with methicillin-resistant Staphylococcus aureus bloodstream infection: results from National Surveillance in Canada, 2008-2012. Infect Control Hosp Epidemiol. 2016;37(4):390-7.

4. Pastagia M, Kleinman LC, de la Cruz EGL, et al. Predicting risk for death from MRSA bacteremia. Emerg Infect Dis. 2012;18(7):1072-80.

5. López-Cortés LE, Del Toro MD, Gálvez-Acebal J, et al. Impact of an evidence-based bundle intervention in the quality-of-care management and outcome of Staphylococcus aureus bacteremia. Clin Infect Dis. 2013;57(9):1225-33.

6. Gudiol F, Aguado JM, Almirante B, et al. Diagnosis and treatment of bacteremia and endocarditis due to Staphylococcus aureus. A clinical guideline from the Spanish Society of Clinical Microbiology and Infectious Diseases (SEIMC). Enferm Infecc Microbiol Clin. 2015;33(9):625.e1-625.e23.

7. Bidell MR, Patel N, O'Donnell JN. Optimal treatment of MSSA bacteraemias: a meta-analysis of cefazolin versus antistaphylococcal penicillins. J Antimicrob Chemother. 2018;73(10):2643-51.

8. García-de-la-Mària $\mathrm{C}$, Gasch $\mathrm{O}$, García-Gonzalez J, et al. The combination of daptomycin and fosfomycin has synergistic, potent, and rapid bactericidal activity against methicillin-resistant Staphylococcus aureus in a rabbit model of experimental endocarditis. Antimicrob Agents Chemother. 2018;62(6):e02633-e2717.

9. Noel AR, Attwood M, Bowker KE, et al. The pharmacodynamics of minocycline alone and in combination with rifampicin against Staphylococcus aureus studied in an in vitro pharmacokinetic model of infection. J Antimicrob Chemother. 2021;76(7):1840-4.

10. Grillo S, Cuervo G, Carratalà J, et al. Impact of betalactam and daptomycin combination therapy on clinical outcomes in methicillin-susceptible Staphylococcus aureus bacteremia: a propensity score- matched analysis. Clin Infect Dis. 2019;69(9): $1480-8$.

11. Tremblay S, Lau TT, Ensom MH. Addition of rifampin to vancomycin for methicillin-resistant Staphylococcus aureus infections: what is the evidence? Ann Pharmacother. 2013;47(7-8):1045-54.

12. Rieg S, Joost I, Weiß V, et al. Combination antimicrobial therapy in patients with Staphylococcus aureus bacteraemia-a post hoc analysis in 964 prospectively evaluated patients. Clin Microbiol Infect. 2017;23(6):406.e1-406.e8.

13. Thwaites GE, Scarborough M, Szubert A, et al. Adjunctive rifampicin for Staphylococcus aureus bacteraemia (ARREST): a multicentre, randomised, double-blind, placebo-controlled trial. Lancet. 2018;391(10121):668-78.

14. Tong SYC, Lye DC, Yahav D, et al. Effect of vancomycin or daptomycin with vs without an antistaphylococcal beta-lactam on mortality, bacteremia, relapse, or treatment failure in patients with MRSA bacteremia: a randomized clinical trial. JAMA. 2020;323(6):527-37.

15. Pujol M, Miró JM, Shaw E, et al. Daptomycin plus fosfomycin versus daptomycin alone for methicillin-resistant Staphylococcus aureus bacteremia and endocarditis: a randomized clinical trial. Clin Infect Dis. $2021 ; 72(9): 1517-25$.

16. Geriak M, Haddad F, Rizvi K, et al. Clinical data on daptomycin plus ceftaroline versus standard of care monotherapy in the treatment of methicillin-resistant Staphylococcus aureus bacteremia. Antimicrob Agents Chemother. 2019;63(5):e02483-18.

17. Ma H, Cheng J, Peng L, et al. Adjunctive rifampin for the treatment of Staphylococcus aureus bacteremia with deep infections: a meta-analysis. PLoS ONE. 2020;15(3):e0230383.

18. Wang C, Ye C, Liao L, et al. Adjuvant beta-lactam therapy combined with vancomycin or daptomycin for methicillin-resistant Staphylococcus aureus bacteremia: a systematic review and meta-analysis. Antimicrob Agents Chemother. 2020;64(11): e01377-20.

19. Kale-Pradhan PB, Giuliano C, Jongekrijg A, et al. Combination of vancomycin or daptomycin and beta-lactam antibiotics: a meta-analysis. Pharmacotherapy. 2020;40(7):648-58.

20. Higgins JP, Altman DG, Gøtzsche PC, et al. The Cochrane collaboration's tool for assessing risk of bias in randomised trials. BMJ. 2011;343:d5928. 
21. Higgins JP, Thompson SG, Deeks JJ, et al. Measuring inconsistency in meta-analyses. BMJ. 2003;327(7414):557-60.

22. Higgins JP, Green S. Cochrane handbook for systematic reviews of interventions. Chichester: Wiley; 2011.

23. Egger M, Davey Smith G, Schneider M, et al. Bias in meta-analysis detected by a simple, graphical test. BMJ. 1997;315(7109):629-34.

24. Cheng MP, Lawandi A, Butler-Laporte G, et al. Adjunctive daptomycin in the treatment of methicillin-susceptible Staphylococcus aureus bacteremia: a randomized controlled trial. Clin Infect Dis. 2021;72(9):e196-203.

25. Davis JS, Sud A, O'Sullivan MVN, et al. Combination of vancomycin and beta-lactam therapy for methicillin-resistant Staphylococcus aureus bacteremia: a pilot multicenter randomized controlled trial. Clin Infect Dis. 2016;62(2):173-80.

26. Fowler VG Jr, Boucher HW, Corey GR, et al. Daptomycin versus standard therapy for bacteremia and endocarditis caused by Staphylococcus aureus. N Engl J Med. 2006;355(7):653-65.

27. Ruotsalainen E, Järvinen A, Koivula I, et al. Levofloxacin does not decrease mortality in Staphylococcus aureus bacteraemia when added to the standard treatment: a prospective and randomized clinical trial of 381 patients. J Intern Med. 2006;259(2):179-90.

28. Ribera E, Gómez-Jimenez J, Cortes E, et al. Effectiveness of cloxacillin with and without gentamicin in short-term therapy for right-sided Staphylococcus aureus endocarditis. A randomized, controlled trial. Ann Intern Med. 1996;125(12):969-74.

29. Levine DP, Fromm BS, Reddy BR. Slow response to vancomycin or vancomycin plus rifampin in methicillin-resistant Staphylococcus aureus endocarditis. Ann Intern Med. 1991;115(9):674-80.

30. Van der Auwera P, Klastersky J, Thys JP, et al. Double-blind, placebo-controlled study of oxacillin combined with rifampin in the treatment of staphylococcal infections. Antimicrob Agents Chemother. 1985;28(4):467-72.

31. Van der Auwera P, Meunier-Carpentier F, Klastersky J. Clinical study of combination therapy with oxacillin and rifampin for staphylococcal infections. Rev Infect Dis. 1983;5(Suppl 3):S515-22.

32. Korzeniowski O, Sande MA. Combination antimicrobial therapy for Staphylococcus aureus endocarditis in patients addicted to parenteral drugs and in nonaddicts: a prospective study. Ann Intern Med. 1982;97(4):496-503.

33. Abrams B, Sklaver A, Hoffman T, et al. Single or combination therapy of staphylococcal endocarditis in intravenous drug abusers. Ann Intern Med. 1979;90(5):789-91.

34. Liu C, Bayer A, Cosgrove SE, et al. Clinical practice guidelines by the infectious diseases society of America for the treatment of methicillin-resistant Staphylococcus aureus infections in adults and children. Clin Infect Dis. 2011;52(3):e18-55.

35. Holland TL, Arnold C, Fowler VG Jr. Clinical management of Staphylococcus aureus bacteremia: a review. JAMA. 2014;312(13):1330-41.

36. Falagas ME, Matthaiou DK, Bliziotis IA. The role of aminoglycosides in combination with a beta-lactam for the treatment of bacterial endocarditis: a metaanalysis of comparative trials. J Antimicrob Chemother. 2006;57(4):639-47.

37. Russell CD, Lawson McLean A, Saunders C, et al. Adjunctive rifampicin may improve outcomes in Staphylococcus aureus bacteraemia: a systematic review. J Med Microbiol. 2014;63(Pt 6):841-8.

38. Dilworth TJ, Ibrahim O, Hall $\mathrm{P}$, et al. $\beta$-Lactams enhance vancomycin activity against methicillinresistant Staphylococcus aureus bacteremia compared to vancomycin alone. Antimicrob Agents Chemother. 2014;58(1):102-9.

39. Casapao AM, Jacobs DM, Bowers DR, et al. Early administration of adjuvant $\beta$-lactam therapy in combination with vancomycin among patients with methicillin-resistant Staphylococcus aureus bloodstream infection: a retrospective multicenter analysis. Pharmacotherapy. 2017;37(11):1347-56.

40. Jorgensen SCJ, Zasowski EJ, Trinh TD, et al. Daptomycin plus $\beta$-lactam combination therapy for methicillin-resistant Staphylococcus aureus bloodstream infections: a retrospective, comparative cohort study. Clin Infect Dis. 2020;71(1):1-10.

41. Zasowski EJ, Trinh TD, Atwan SM, et al. The impact of concomitant empiric cefepime on patient outcomes of methicillin-resistant Staphylococcus aureus bloodstream infections treated with vancomycin. Open Forum Infect Dis. 2019;6(7):ofz077.

42. Alosaimy S, Sabagha NL, Lagnf AM, et al. Monotherapy with vancomycin or daptomycin versus combination therapy with $\beta$-lactams in the treatment of methicillin-resistant Staphylococcus aureus bloodstream infections: a retrospective cohort analysis. Infect Dis Ther. 2020;9(2):325-39. 
43. Dhand A, Bayer AS, Pogliano J, et al. Use of antistaphylococcal betalactams to increase daptomycin activity in eradicating persistent bacteremia due to methicillin-resistant Staphylococcus aureus: role of enhanced daptomycin binding. Clin Infect Dis. 2011;53(2):158-63.

44. See EJ, Jayasinghe K, Glassford N, et al. Long-term risk of adverse outcomes after acute kidney injury: a systematic review and meta-analysis of cohort studies using consensus definitions of exposure. KidneyInt. 2019;95(1):160-72.

45. Rose W, Fantl M, Geriak M, et al. Current paradigms of combination therapy in methicillin-resistant Staphylococcus aureus (MRSA) bacteremia: Does it work, which combination and for which patients? Clin Infect Dis. 2021. https://doi.org/10.1093/cid/ ciab452

46. McCreary EK, Kullar R, Geriak M, et al. Multicenter cohort of patients with methicillin-resistant Staphylococcus aureus bacteremia receiving daptomycin plus ceftaroline compared with other MRSA treatments. Open Forum Infect Dis. 2019;7(1):ofz538.

47. Ahmad O, Crawford TN, Myint T. Comparing the outcomes of ceftaroline plus vancomycin or daptomycin combination therapy versus monotherapy in adults with complicated and prolonged methicillin-resistant Staphylococcus aureus bacteremia initially treated with supplemental ceftaroline. Infect Dis Ther. 2020;9(1):77-87.

48. Johnson TM, Molina KC, Miller MA, et al. Combination ceftaroline and daptomycin salvage therapy for complicated methicillin-resistant Staphylococcus aureus bacteraemia compared with standard of care. Int J Antimicrob Agents. 2021;57(4):106310.

49. Hassoun A, Linden PK, Friedman B. Incidence, prevalence, and management of MRSA bacteremia across patient populations-a review of recent developments in MRSA management and treatment. Crit Care. 2017;21(1):211.

50. Alosaimy S, Lagnf AM, Morrisette T, et al. Standardized treatment and assessment pathway improves mortality in adults with methicillin-resistant Staphylococcus aureus bacteremia: STAPH study. Open Forum Infect Dis. 2021;8(7):ofab261.

51. Weis S, Kesselmeier M, Davis JS, et al. Cefazolin versus anti-staphylococcal penicillins for the treatment of patients with Staphylococcus aureus bacteraemia. Clin Microbiol Infect. 2019;25(7):818-27.

52. Eljaaly K, Alshehri S, Erstad BL. Systematic review and meta-analysis of the safety of antistaphylococcal penicillins compared to cefazolin. Antimicrob Agents Chemother. 2018;62(4):e01816-e1817. 